\title{
Optimal operation of a hybrid-energy microgrid with energy storage system
}

\author{
Mohamed Elgamal ${ }^{1,2,}{ }^{*}$, Nikolay $V$. Korovkin ${ }^{l}$, Ahmed Refaat $^{l}$ and Akram Elmitwally ${ }^{2}$ \\ 1 Peter the Great St.Petersburg Polytechnic University, Polytechnicheskaya, 29, St. Petersburg, \\ 195251, Russia \\ ${ }^{2}$ Mansoura University, Elgomhouria St., Mansoura City, 35516, Egypt.
}

\begin{abstract}
In this paper, a day-ahead profit-maximizing energy management scheme for a grid-tied microgrid operation is proposed. The microgrid contains various types of distributed energy resources (DERs) and an inverter-interfaced battery-bank storage system. The average of day-ahead hourly forecasted data for loads, wind speed, and solar radiation are inputted into the framework of energy management (EMF). To optimize the microgrid performance, EMF determines the hourly dispatch of reactive and active power for each DER. Also, it specifies the discharging and charging times of the energy storage system and the onload tap changer position setting of the transformer connected to the main grid. The main aim is to maximize the revenue of microgrid meeting all technical limitations. The main grid can sell/buy reactive and active powers to/from the microgrid with a variable daily energy price of the market. A collective rule base-BAT algorithm is implemented as a solver of the energy management optimization problem for a grid-tided microgrid. Furthermore, the ability of the suggested EMF is proved in comparison with recent approaches.
\end{abstract}

\section{Introduction}

Microgrid (MG) provides promising solutions to the increase in the prices of traditional power sources, environmental concerns, and operational efficiency. It is a distribution grid integrating DERs to feed loads in isolated or grid-connected mode [1-3]. Different DERs in the MG must be managed in a synchronized way. Therefore, an Energy Management Framework (EMF) has to be developed [4]. EMF is a computer system that provides the functions necessary for the secure dynamic operation of electrical power system at minimum cost [5]. It implements a set of jobs for instance supervising, managing, and cooperating, and controlling. EMF may also forecast generating energies, demand, and energy tariffs of the market. So, it aims to optimize MG performance considering the techno-economic restrictions [4].

The EMF of MG can be classified into three types, namely, centralized, distributed, and hierarchical EMFs [5]. For the centralized EMF, the fundamental supervisor combines whole data of the system like characteristics of DERs, load power, and running costs.

\footnotetext{
${ }^{*}$ Corresponding author: elgamal.mm@edu.spbstu.ru
} 
Afterwards, it finds the optimal energy management results of $\mathrm{MG}$ and sends these decisions to all local controllers. For the hierarchical EMF, the MG supervisor interchanges the data with its observers. The main supervisor of $\mathrm{MG}$ determines the best evaluation and sends it back to the whole observers. The great merit of hierarchical and centralized approaches is that it obtains the best solution value accurately, especially for small systems.

The popular methods applied in centralized EMF contain artificial intelligence methods, particle swarm optimization (PSO), Petri-net method, BAT algorithm, mathematical programming, hyper-spherical search algorithm [6-24]. Refs. [7-24] provide optimization methods based-EMFs for a grid connected-MG to reduce the running cost and the power loss. These only contain the day-ahead scheduling and dispatch of active power of the DERs. Therefore, this will decrease the MG revenue, and increase the power loss. It may also cause voltage abuses and overload conditions. Ref. [6] proposed an EMF based on PSO that included the day-ahead scheduling and dispatch of active and reactive power of the dispatchable DERs. Nevertheless, it neglected the utilization of typical supporting equipment like as shunt capacitor banks and on-load tap changer (OLTC).

In this paper, a day-ahead profit-maximizing EMF based on BAT method for a grid-tied $\mathrm{MG}$ operation is formulated. The MG has photovoltaic generators (PVG), wind turbine generators (WTG), microturbine generator (MTG), a fuel cell generator (FCG), and an energy storage system (ESS). The proposed EMF have the next advantages:

- It integrates a rule base into the BAT algorithm to lower the computation time.

- Considers the dispatch of reactive power among the dispatchable DERs.

- Includes the active and reactive power interchange between the main power grid and the MG.

- Keeps voltages of buses, removes lines overloads, and avoids burden on the OLTC.

\section{Solution Algorithm}

In this work, dispatchable DERs and ESS are successfully managed to maximize the MG profits. The active and reactive powers are included together and a proper cost is included for each. The profit of $\mathrm{MG}$ at an hour $\mathrm{t}\left(P R O_{M G, t}\right)$ is expressed as:

$$
P R O_{M G, t}=\left[\beta_{P, b, t} P_{t}+\beta_{Q, b, t} Q_{t}\right]-\left[O C_{t}+M C_{t}\right]
$$

The first two element in (1) denote the hourly income from the sale of reactive and active power to the main power grid and the consumers loads, respectively. The following two elements denote the total hourly costs of operation and maintenance for DERs and ESS, respectively.

$\beta_{P, b, t}$ and $P_{t}$ represent the buying price of active power from the market $(\$ / \mathrm{kWhr})$ and the magnitude of hourly active power purchased from the main grid ( $k W h r)$, respectively. $\beta_{Q, b, t}$ and $Q_{t}$ represent the buying price of reactive power from the market ( $\$ / k V A r h r$ ) and the magnitude of hourly reactive power purchased from the main grid (kVArhr), respectively.

The total operating cost $\left(O C_{t}\right)$ at the time $\mathrm{t}$ can be calculated using the following equation:

$$
O C_{t}=C_{g, t}+\sum_{i=1}^{n_{E S S}} C_{E S S, t}^{i}+\sum_{j=1}^{n_{d g}} C_{d g, t}^{j}
$$

Where, $C_{g, t}, C_{E S S, t}, C_{d g, t}$ are operating costs at time $t$ for main grid, the $i^{t h}$ ESS unit, the $j^{\text {th }}$ DER unit respectively.

The hourly operating cost of the energy delivered by the main grid in a corresponding control time $\left(C_{g, t}\right)$ can be modeled as: 


$$
\begin{gathered}
C_{P, g, t}= \begin{cases}\beta_{P, b, t} P_{g, t} & \text { if } P_{g, t}>0 \\
\beta_{P, s, t} P_{g, t} & \text { if } P_{g, t}<0 \\
0 & \text { if } P_{g, t}=0\end{cases} \\
C_{Q, g, t}= \begin{cases}\beta_{Q, b, t} Q_{g, t} & \text { if } Q_{g, t}>0 \\
\beta_{Q, s, t} Q_{g, t} & \text { if } Q_{g, t}<0 \\
0 & \text { if } Q_{g, t}=0\end{cases} \\
\beta_{P, s, t}=(1-\lambda) \beta_{P, b, t} \\
\beta_{Q, s, t}=(1-\lambda) \beta_{Q, b, t} \\
C_{g, t}=C_{P, g, t}+C_{Q, g, t}
\end{gathered}
$$

Where, $\beta_{P, s, t}, \beta_{Q, s, t}$ are the prices of active and reactive powers sold by the MG to the main grid, $P_{g, t}, Q_{g, t}$ are active and reactive powers of main grid at time $t$, and $\lambda$ is tax rate of power sold of main grid, $\lambda$ is selected as $10 \%$ in this study [12]. The hourly operating cost of the ESS is modeled according to system efficiency [9]. [19]:

The operation cost of each controllable DER $\left(C_{d g, t}\right)$ at the time $t$ is calculated as [6],

$$
C_{d g, t}=\beta_{P, t} P_{d g, t}+\beta_{Q, t} Q_{d g, t}
$$

Where, $\beta_{P, t}, \beta_{Q, t}$ are the operating costs of the generation of active power $\left(P_{d g, t}\right)$ and generation of reactive power $\left(Q_{d g}, t\right)$ by the DER at the hour $\mathrm{t}$.

The objective is

$$
\text { Maximize } \quad \sum_{t=1}^{24} P R O_{M G, t}
$$

The MG operation problem is formulated as a constrained optimization problem as provided in $[6,25]$. The BAT technique is a recent metaheuristic optimization solver presented in [12]. A rule base-assisted BAT is employed to solve the above formulated optimization problem. The control variables of the optimization problem formulated in section 2 above are the hourly-average reactive and active powers of DERs, OLTC tap position, and ESS charging and discharging powers. The proposed algorithm is depicted in the following steps.

1. For an hour $t$, read the MG data.

2. Initialize and update the iteration counter.

3. Initialize the bat population, for each bat $b$ choose initial position vector $x_{b, 0}$ (initial values of the control variables vector).

4. For each hour, each bat in the population adjusts the charging and discharging mode of ESS, using the next rules:

a) If the purchasing price of active power from the market $\left(\beta_{P, b, t}\right)$ is smaller than the limit of ESS charging price ( 0.4 of the highest purchasing price of active power from the market [26]) then bat sets the ESS mode for charging state and produces a positive random amount of the ESS power in this range $\left\{P_{C h, \max }, 0\right\}$, where $P_{C h, \max }$ is the maximum ESS power of charge.

b) If the purchasing price of active power from the market $\left(\beta_{P, b, t}\right)$ is above the limit of ESS charging price, and ESS state of charge at time $t\left(S O C_{t}\right)$ is less than the maximum limit $\left(S O C_{\max }\right)$, and the full renewable DERs power generation is greater than the full MG load power, then the bat sets the mode of ESS as a charging mode and adjusts the power of ESS as a positive random amount in the range of 
difference between the full renewable DERs power generation and the total demand load of MG.

c) If the sale price of active power of the market $\left(\beta_{P, s, t}\right)$ is above the ESS discharging price limit ( 0.7 of the highest sale price of active power of the market [26]) and $S O C_{t}$ is less than the minimum limit $\left(S O C_{\min }\right)$, then the bat sets the mode of ESS for discharging and produces a negative random amount of the ESS power in this range $\left\{0, P_{D i s, \max }\right\}$, where $P_{D i s, \max }$ is the maximum ESS power of discharge .

5. Implement load flow analysis. Subsequently, determine the value of objective function using (1) for each bat, and set it as the best solution of this bat. Check all constraints [25]. If a constraint is violated, the corresponding bat is excluded by high penalty. Then, determine the global best solution (Gbest) of the bat population by comparing the individuals' local best solution of all successful bats.

6. Update the velocity and the position of each bat as given in [12].

7. Modify the current position and local optimal solution of each bat using random walk as in [12].

8. Increase iteration number by 1 . If iteration number is larger than the maximum number of iterations, then save results for this day hour and go to step 9. Otherwise, go to step 5 .

9. Consider the next day hour and go to step 1 until all hours are done.

Fig. 1 reveals the flowchart of the solution algorithm.

\section{Simulation Model}

Fig.2 provides the well-known IEEE 33-bus distribution network [27], is utilized as a gridtided MG to assess the proposed algorithm. As depicted in Fig.2, WTGs are set at nodes 16, $21,25,32$. PVGs are set at nodes 12, 19, 23, 29. MTG is set at node 26. FCG is set at node 9. ESS is set at node 5. Table 1 provides the generation limits for each unit. The suggested approach is modeled as a script-file in the MATLAB environment and implemented using a personal computer with 2.3-GHz processor and 4-GB RAM. The full ESS data and operation costs for MTG and FCG are given in $[6,24,28]$. Technical maintenance charges per year for MTG, FCG, ESS, WTG. and PVG are offered in $[6,12]$.

Table 1. Generation limits for each generation unit

\begin{tabular}{|l|c|c|c|c|c|}
\hline \multirow{2}{*}{ Unit } & \multirow{2}{*}{ Location } & \multicolumn{2}{|c|}{ Active power $(\mathrm{KW})$} & \multicolumn{2}{c|}{ Reactive power (KVAr) } \\
\cline { 3 - 6 } & & $\mathrm{P}_{\max }$ & $\mathrm{P}_{\min }$ & $\mathrm{Q}_{\max }$ & $\mathrm{Q}_{\min }$ \\
\hline $\mathrm{PVG}_{1}$ & 12 & 350 & 0 & 0 & 0 \\
\hline $\mathrm{PVG}_{2}$ & 19 & 450 & 0 & 0 & 0 \\
\hline $\mathrm{PVG}_{3}$ & 23 & 350 & 0 & 0 & 0 \\
\hline $\mathrm{PVG}_{4}$ & 29 & 400 & 0 & 0 & 0 \\
\hline $\mathrm{WTG}_{1}$ & 17 & 550 & 0 & 0 & 0 \\
\hline $\mathrm{WTG}_{2}$ & 21 & 1250 & 0 & 0 & 0 \\
\hline $\mathrm{WTG}_{3}$ & 25 & 650 & 0 & 0 & 0 \\
\hline WTG & 32 & 550 & 0 & 0 & 0 \\
\hline FCG & 9 & 1000 & 150 & 750 & 0 \\
\hline MTG & 26 & 750 & 112.5 & 520 & 0 \\
\hline ESS & 5 & 1500 & -1500 & 0 & 0 \\
\hline
\end{tabular}




\section{$4 \quad$ Results and Discussion}

\subsection{Simulation Results of the proposed algorithm}

The solar radiation and wind speed values are given in [25] for a normal day during the summer. The buying prices of active and reactive powers from the market are reported in $[9,29]$. The hourly mean of powers of WTG, PVG and the demand load of MG are reported in [25] and are depicted in Fig.3. Suppose that the beginning level of ESS charge is $25 \%$ and the load powers on nodes from bus- 1 to bus- 6 raise to nearly $300 \%$ of their normal powers. As expected, ESS will charge from the bulk-power system and begin this state from nearly midnight 02:00 to the early morning 07:00, through low price and off-peak demand load time, as depicted in Fig.4. Also as revealed in Fig.4, FCG and MTG powers are set optimally to maximize MG profit. The profit of $M G$ of the selected day in the summer season is 5761 \$. It is worth mentioning that the MG profit of the selected day will be decreased to 5121 \$ if the ESS is not used. The daily OLTC tap position change is shown in Fig.5. The daily OLTC operations is maintained below 7 operations as a practical limit.

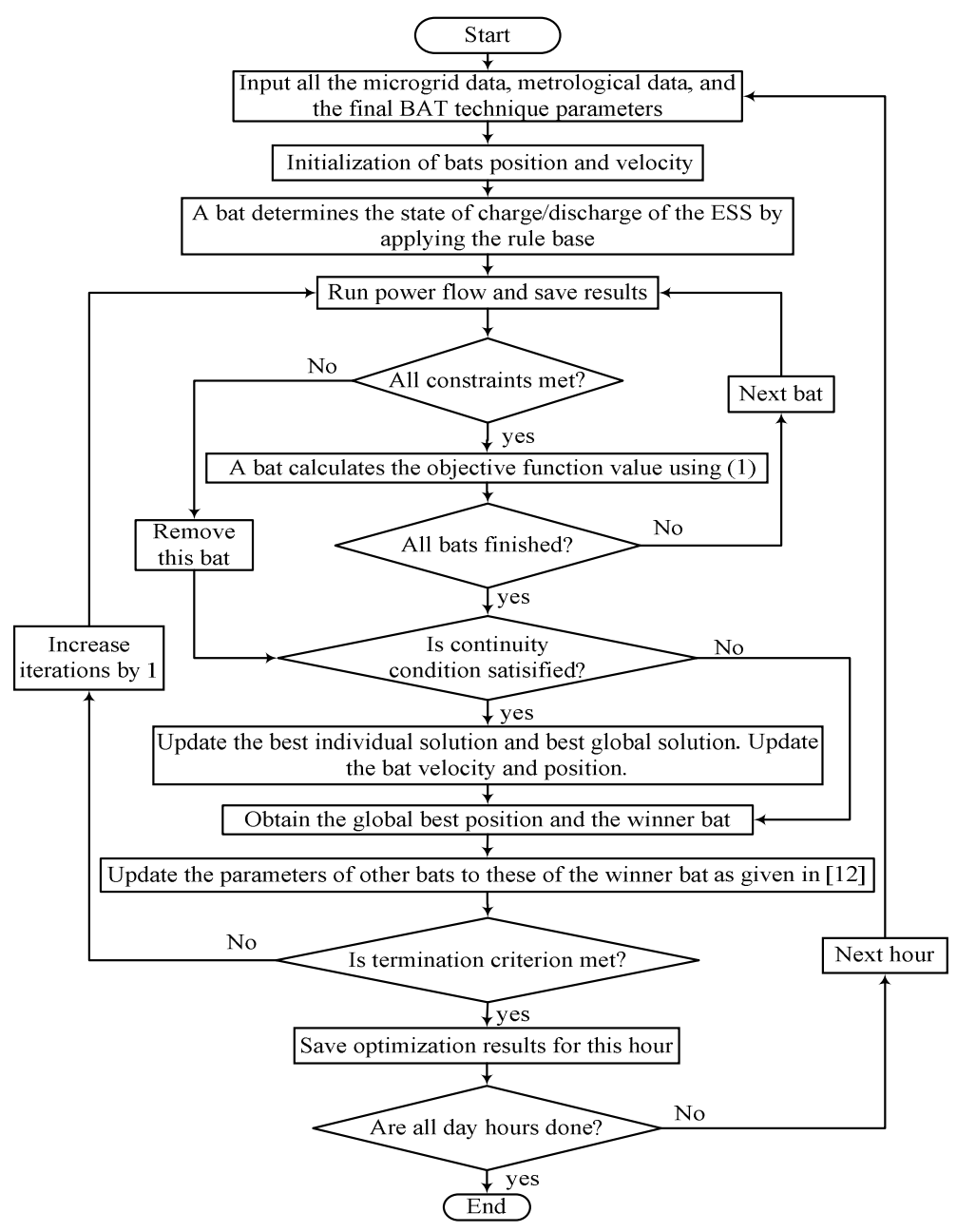

Fig.1. Flowchart of solution algorithm 


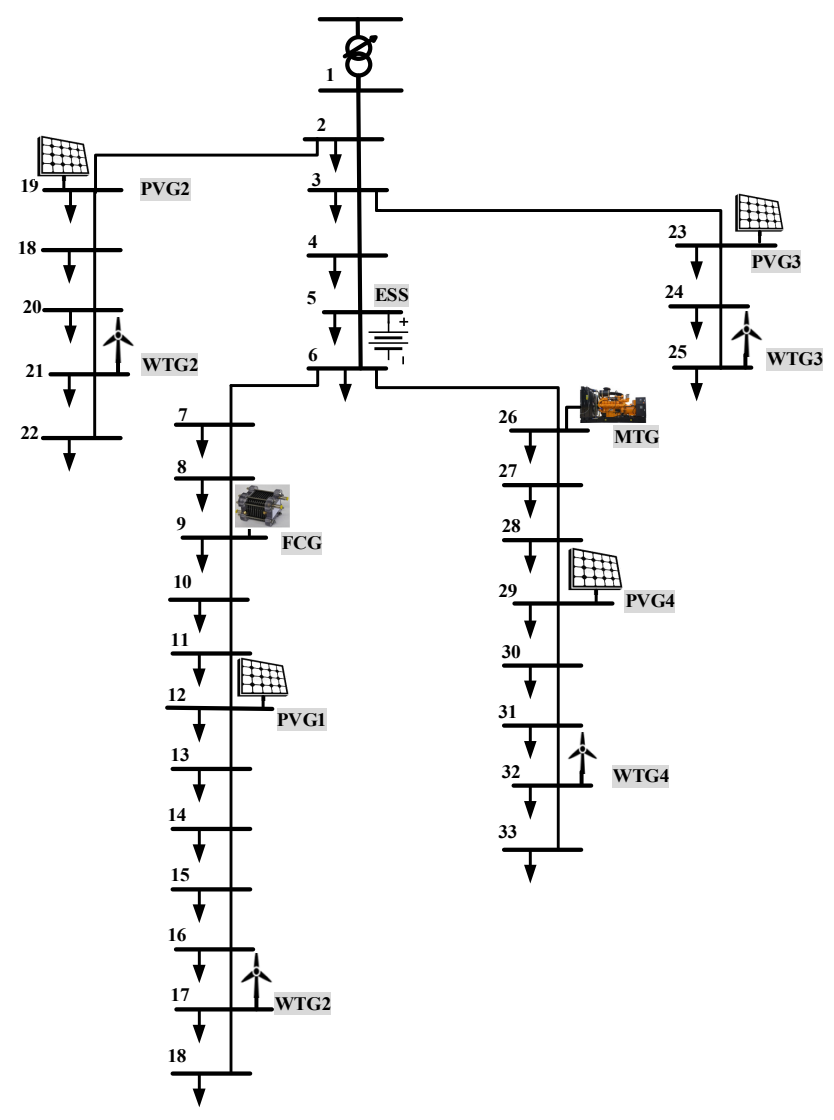

Fig. 2. The proposed grid-tided MG

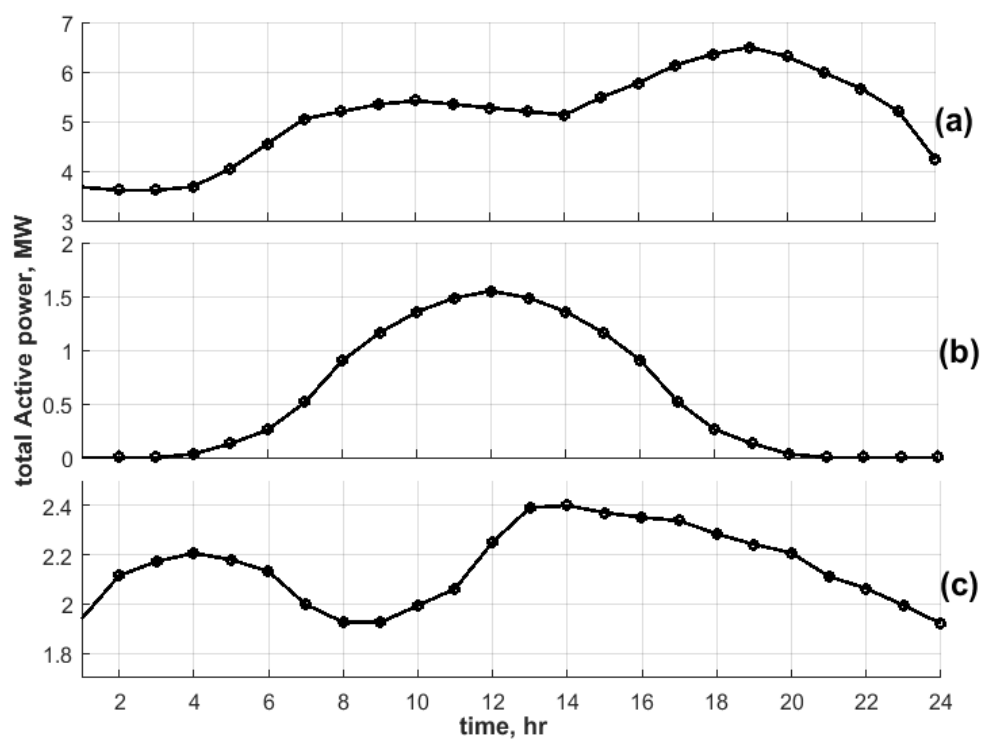

Fig. 3. Hourly average active power of total demand load, total PVG units, and total WTG units 


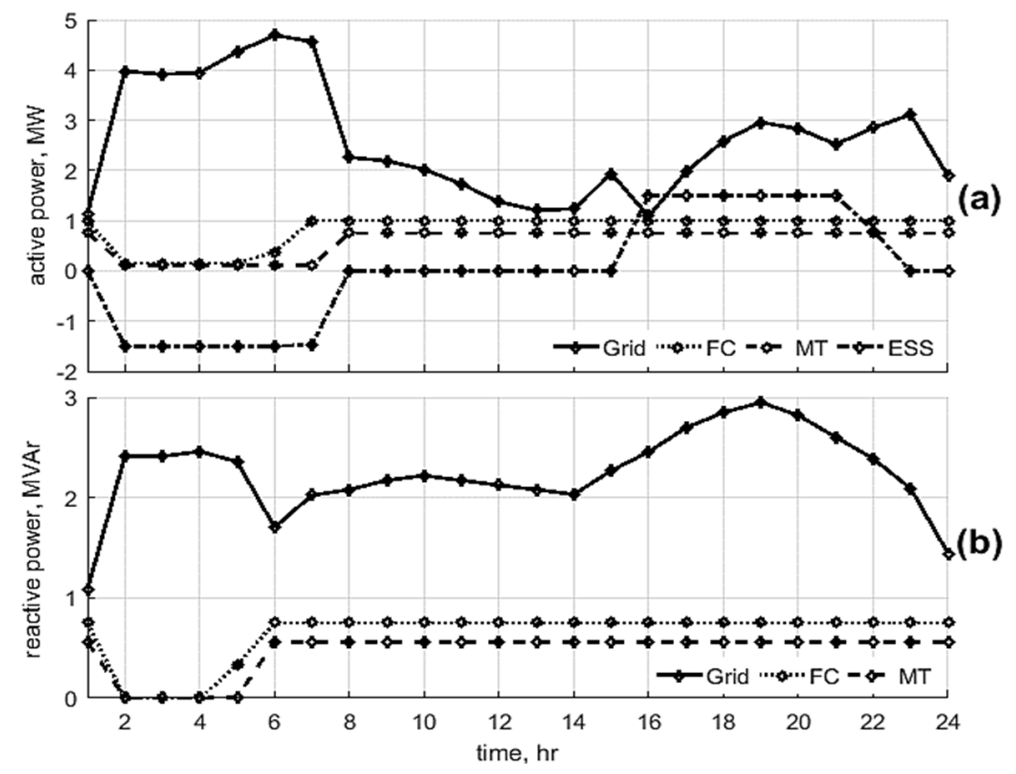

Fig. 4. Power setting of ESS, MTG, FCG, and main grid.

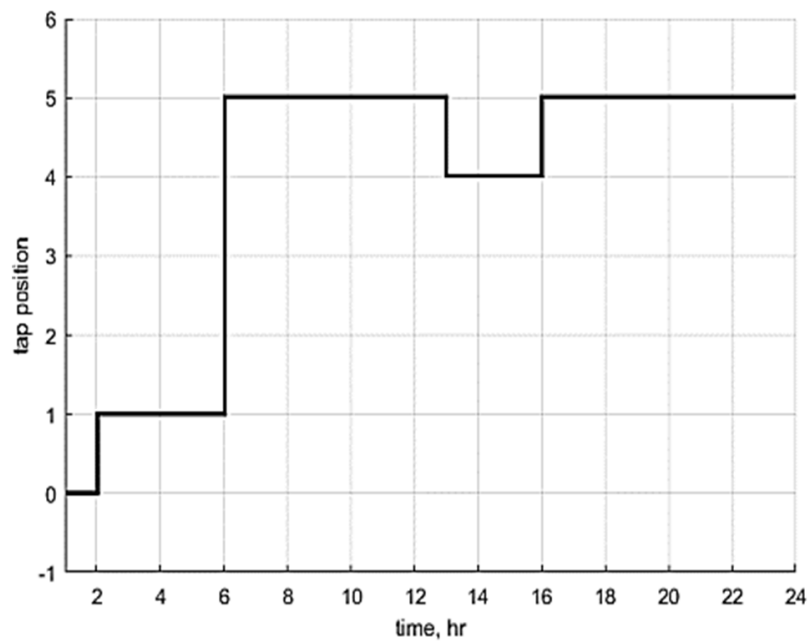

Fig. 5. Tap setting of OLTC.

\subsection{Comparison of performance}

Table 2 provides a comparison to evaluate the effectiveness of the proposed algorithm against recent approaches presented in [6] and [12]. It is worth mentioning that the proposed method attains the most excellent economic performance. From the technical aspect, it maintains the maximum and minimum voltage for all buses within the acceptable limits, however voltage problems occur using the approach in [12]. Also, it maintains lines power flow under the allowable maximum capacities of lines, while an overload occurs by the strategy in [6]. Line 1-2 suffers from an overload during on-peak hours from 18:00 to 20:00 and from 22:00 to 23:00. In addition, Fig. 6 shows state of charge of the ESS using the proposed algorithm and recent approaches presented in [6] and [12]. Fig. 7 shows the state of convergence of these approaches at $t=07: 00$. As shown in Fig. 7, it is noted that 
the proposed approach has the best convergence accuracy and rate. It can be deduced that the proposed strategy is more effective, successful, and more accurate than approaches presented in [6] and [12].

Table 2. Daily performance evaluation of operating methods

\begin{tabular}{|l|l|l|l|}
\hline Item & Proposed strategy & Ref. [12] & Ref. [6] \\
\hline MG profit, \$ & 5761 & 5415.8 & 5730.7 \\
\hline Mean active power loss, $\mathrm{kW}$ & 70.3 & 111.16 & 74.36 \\
\hline Lowest bus voltage, p.u. & 0.952 & 0.937 & 0.95 \\
\hline Highest bus voltage, p.u. & 1.045 & 1.014 & 1.021 \\
\hline Computation time, sec & 1669 & 1770 & 1923 \\
\hline
\end{tabular}

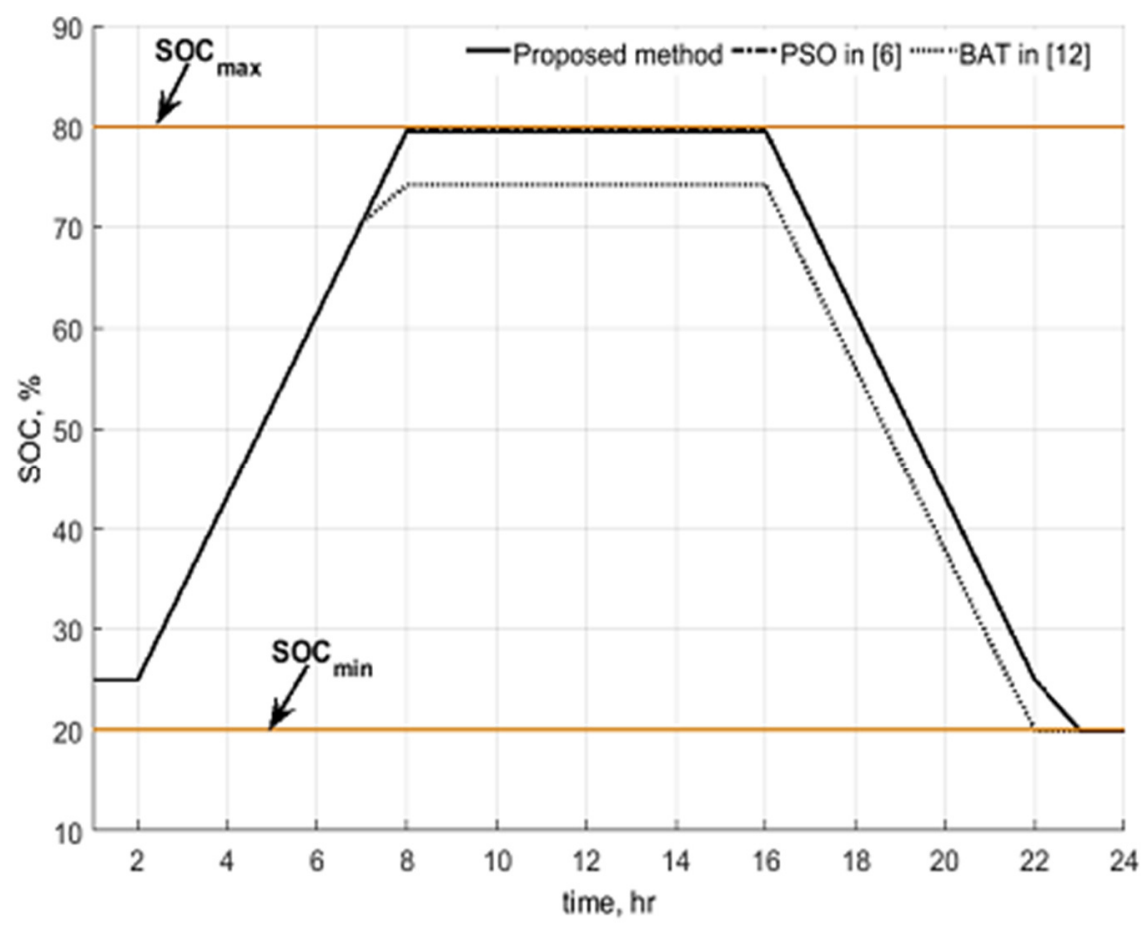

Fig. 6. SOC of ESS by the proposed algorithm, Ref. [12] and Ref. [6] 


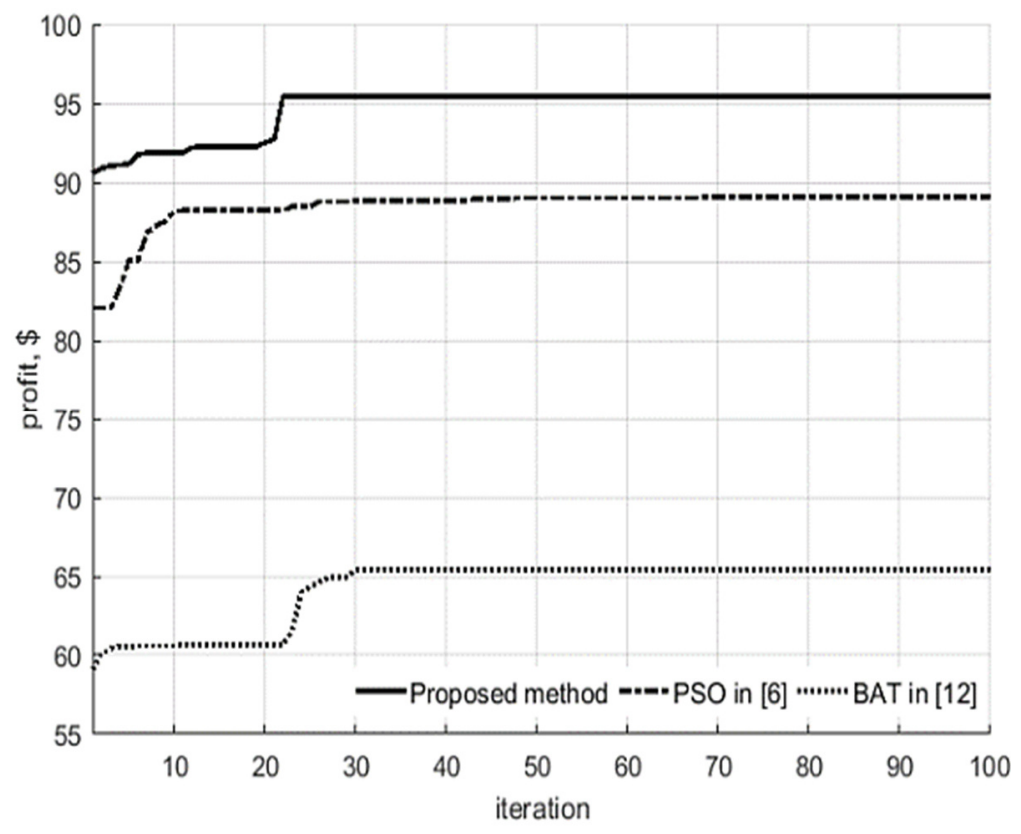

Fig. 7. Sate of convergence by the proposed algorithm, Ref. [12] and Ref. [6] at the $7^{\text {th }}$ hour.

\section{Conclusions}

The paper presents a day-ahead profit-maximizing energy management strategy of a gridtied MG. The MG contains hybrid renewable energy resources, fuel cell, microturbine, and a battery-bank ESS. Maximum possible power is extracted from PVGs and WTGs. Hence, they are treated as non-dispatchable DERs with unity power factor. The main grid exchanges both reactive and active powers with the microgrid at a variable daily energy price of the market. The forecasted day-ahead hourly average of solar insolation, wind speed, and MG demand load are inputted into the EMF. Consequently, it determines the hourly dispatch of active and reactive power for each dispatchable DERs. Also, EMF specifies the discharging and charging state and power of the ESS. Besides, the EMF controls the position setting of the on-load tap changer and protects it from overstresses. The main aim is to maximize the income of the microgrid meeting all technical constraints. A collective rule base-BAT technique is used as a solver for the day-ahead energy management optimization problem of a grid-tided microgrid. Furthermore, the aptness of the presented EMF is confirmed by comparing efficacy with modern approaches.

\section{References}

1. A. Micallef, M. Apap, C. Spiteri-Staines, and J. M. Guerrero, IEEE Trans. Smart Grid, 6, 6, 2736-2745, (2015).

2. N. Hatziargyriou, H. Asano, R. Iravani, and C. Marnay, IEEE Power and Energy Magazine, 5, 4. 78-94, (2007).

3. W. Su, J. Wang, and J. Roh, IEEE Trans. Smart Grid, 5, 4, 1876-1883, (2014).

4. C. Chen, S. Duan, T. Cai, B. Liu, and G. Hu, IET Renew. Power Gener., 5, 3, 258, (2011).

5. F. Nejabatkhah and Y. W. Li, IEEE Transactions on Power Electronics, 30, 12. 7072-7089, (2015). 
6. H. Saboori and R. Hemmati, Renew. Sustain. Energy Rev., 71, 11, 365-372, (2017).

7. P. Chaweewat, J. G. Singh, W. Ongsakul, and A. K. Srivastrava, Proceedings of the 2016 International Conference on Cogeneration, Small Power Plants and District Energy, ICUE 2016, (2016)

8. D. E. Olivares, C. A. Canizares, and M. Kazerani, IEEE Trans. Smart Grid, 5, 4, 1864-1875, (2014).

9. Y. Wu, V.K.N. Lau, D.H.K. Tsang, L.P. Qian, and L. Meng, IEEE Syst. J., 8, 2, 562-576, (2014).

10. A. Sheikhi, M. Rayati, S. Bahrami, and A. M. Ranjbar, IEEE Trans. Smart Grid, 6, 2, 675-683, (2015).

11. C. O. Adika and L. Wang, IEEE Trans. Smart Grid, 5, 2, 673-682, (2014).

12. J. Han, C. Choi, W. Park, I. Lee, and S. Kim, IEEE Trans. Consum. Electron., 60, 2, 198-202, (2014).

13. C. F. Calvillo, A. Sánchez-Miralles, J. Villar, and F. Martín, Appl. Energy, 182, 340-357, (2016).

14. A. Berrada and K. Loudiyi, Renew. Sustain. Energy Rev., 59, 1, 1117-1129, (2016).

15. S. A. Alavi, A. Ahmadian, and M. Aliakbar-Golkar, Energy Convers. Manag., 95, 10, 314-325, (2015).

16. M. Sedghi, A. Ahmadian, and M. Aliakbar-golkar, IEEE Trans. Power Syst., 31, 1, 304-316, (2016).

17. J.M. Javadi, M. Sadegh, A. Anvari-Moghaddam, Guerrero, Proc. 7th Sol. Integr. Work. Berlin, Ger., 1-6, (2017).

18. D. Fendri and M. Chaabene, 4th International Conference on Control, Decision and Information Technologies (CoDIT), 0992-0996, (2017).

19. M.J. Sanjari, H. Karami, A. H. Yatim, and G. B. Gharehpetian, Appl. Soft Comput. J., 37, 15-23, (2015).

20. B. Bahmani-Firouzi and R. Azizipanah-Abarghooee, Int. J. Electr. Power Energy Syst., 56, $42-$ 54, (2014).

21. M. Asaduz-Zaman and A. H. Chowdhury, in 2nd International Conference on Electrical Engineering and Information and Communication Technology, iCEEiCT, (2015).

22. N.A. Belyaev, N. V. Korovkin, O. V. Frolov, and V. S. Chudnyi, Russ. Electr. Eng., 84, 2, 74 80, (2013).

23. N.A. Belyaev, N. V. Korovkin, V. S. Chudny, and O. V. Frolov, 2015 IEEE NW Russ. Young Res. Electr. Electron. Eng. Conf., 150-154, (2015).

24. A. Chernavin and N.V. Korovkin, 2016 IEEE NW Russ. Young Res. Electr. Electron. Eng. Conf., 527-529, (2016).

25. M. Elgamal, N.V. Korovkin, A. Refaat, and A. Elmitwally, 2019 IEEE Conf. Russ. Young Res. Electr. Electron. Eng., 489-494, (2019).

26. N.M. Nor, A. Ali, T. Ibrahim, and M.F. Romlie, IEEE Access, 6, 1137-1154, (2017).

27. P. Hu, H. Chen, and X. Zhu, Proceedings of International Conference on Harmonics and Quality of Power, ICHQP, 2016-Decem, 727-732, (2016).

28. A. Refaat, M. Elgamal, and N. V Korovkin, 2019 IEEE Conf. Russ. Young Res. Electr. Electron. Eng., 407-412, (2019).

29. D.T. Nguyen and L.B. Le, IEEE Trans. Smart Grid, 5, 4, 1608-1620, (2014). 